\title{
Exploring the utility of a spontaneous adverse drug reaction reporting system in identifying drug-drug interactions between antiretrovirals, antitubercular drugs, and cotrimoxazole: a case/non-case analysis
}

\author{
Josiah Tatenda Masuka ${ }^{1,2}$ ( Anisa Mosam² $\cdot$ Star Khoza $^{3}$
}

Published online: 22 September 2020

(c) Springer Nature Switzerland AG 2020

\begin{abstract}
Background Drug-drug interactions (DDIs) cause significant morbidity and mortality, especially in patients with HIV with opportunistic infections such as tuberculosis. However, the literature on quantitative signal detection analyses for DDIs within the national spontaneous reporting systems (SRSs) of countries with high HIV/tuberculosis burdens is lacking.

Objective Our objective was to explore the utility of using post-marketing SRSs in quantitative signal detection analyses of DDIs.

Methods A case/non-case analysis using the Zimbabwean adverse drug reaction (ADR) database obtained from VigiBase ${ }^{\circledR}$ was utilized for quantitative signal detection using $2 \times 2$ contingency table calculations. Cases were defined as individual case safety reports (ICSRs) with the ADR of interest, and non-cases included the rest of the ICSRs. The exposure of interest was the use of a drug of interest.

Results Signals of disproportionate reporting (SDRs) were observed for hepatotoxicity for the combined use of highly active antiretroviral therapy (HAART) and antitubercular treatments (ATT) [ADR reporting odds ratio (ROR) 43.78; 95\% CI 5.24-366.08], HAART and isoniazid (ROR 44.84; 95\% CI 5.36-374.99), and isoniazid and nevirapine-based HAART (ROR 35.60; 95\% CI 9.39-134.89). SDRs were also observed for the combined use of nevirapine-based HAART and cotrimoxazole for Stevens-Johnson syndrome (ROR 28.91; 95\% CI 14.00-59.70), severe cutaneous ADRs (ROR 16.10; 95\% CI 9.40-27.57), and rash (OR 2.18; 95\% CI 1.69-2.81).

Conclusion It is feasible to conduct signal detection analyses for DDIs within relatively small SRS databases. However, the observed potential SDR for the respective DDI should be investigated further as the method is only a hypothesis-generation analysis.
\end{abstract}

\section{Introduction}

Drug-drug interactions (DDIs) are defined as the modification of the safety and/or efficacy profile of any medicine concomitantly administered with one or more other drugs

Josiah Tatenda Masuka

josiahmasuka@gmail.com

1 Harare Central Hospital, Southerton, PO Box ST14, Harare, Zimbabwe

2 Department of Dermatology, Nelson R Mandela School of Medicine, University of KwaZulu-Natal, Congella, Private Bag X7, Durban 4013, South Africa

3 Discipline of Pharmacology and Clinical Pharmacy, Faculty of Natural Sciences, School of Pharmacy, University of the Western Cape, Private Bag X17, Bellville 7535, South Africa
[1]. They result from the alteration of the pharmacological effects of one drug because of the actions of the co-administered drug leading to unpredictable and/or predictable clinical effects [2]. DDIs often lead to treatment inefficacy and/ or substantial morbidity [3]. They account for $30 \%$ of all adverse drug reactions (ADRs) $[4,5]$ and between 1 and 5\% of hospital admissions in the general population and elderly populations, respectively [6]. DDIs can be classified as pharmacodynamic, pharmacokinetic, or pharmaceutical interactions, depending on the involved mechanism of action [7].

DDIs are difficult to detect because of their low incidence rates and even lower reporting rates [3, 8, 9]. However, some data mining algorithms have been developed for spontaneous reporting systems (SRSs) to confirm known signals or to flag and ultimately prioritize the clinical review of potential signals of DDIs $[3,8]$. SRS provides a larger pool of patients exposed to a substantial number of co-medications, 
unlike the situation in pre-marketing clinical trials [8]. This improves the detectability of rarer ADRs, such as DDIs, using univariate logistic regression and additive and multiplicative modelling, amongst others [8, 10, 11]. Previous studies have validated the utility of detecting signals of disproportionate reporting (SDR) in SRSs such as VigiBase ${ }^{\circledR}$ or in smaller, national databases $[4,9,12]$. As such, postmarketing surveillance, as exemplified by SRS, can detect rare and long-latency ADRs, unlike randomized controlled trials, which have limited drug co-prescriptions and duration of follow-up [8]. However, SRSs have several limitations, including under-reporting, poor reporting of concomitant medications, and unavailability of denominator data.

Similar approaches may be useful in assessing potential DDI signals with the concurrent use of highly active antiretroviral therapy (HAART) and drugs for the treatment and prevention of opportunistic infections. It has previously been suggested that DDIs between non-nucleotide reverse transcriptase inhibitors (NNRTIs) and rifampicin in resourcelimited settings should be assessed, given the restricted formularies and use of standardized therapies and fixed-dose combinations alongside limited therapeutic drug monitoring services [13, 14]. Moreover, the commonly available NNRTIs, nevirapine and efavirenz, have well-documented overlapping toxicities with rifampicin, which may lead to poor adherence and treatment interruptions $[15,16]$. As it is critically important to provide HAART and antitubercular treatment (ATT) to patients co-infected with HIV/tuberculosis in resource-limited settings to reduce morbidity and mortality, HAART must be compatible with ATT to avoid potential or actual DDIs with rifampicin $[17,18]$. Our group has previously reported on the higher frequency of some ADRs (e.g. hepatic disorders, peripheral neuropathy, and rash) in patients exposed to both antiretrovirals and ATT using spontaneous adverse reaction data [19]. In this study, we explored the utility of using the Zimbabwean post-marketing SRS for quantitative signal detection analyses of potential DDIs between antiretroviral drugs, ATT, and cotrimoxazole. We hypothesised that concurrent use of antiretrovirals and prophylactic medicines for tuberculosis or opportunistic diseases increase the risk of certain ADRs.

\section{Methods}

\section{Setting and design}

We carried out a case/non-case analysis for some preferred terms (PTs) or ADRs of interest in the Zimbabwean SRS database for individual case safety reports (ICSRs) mentioning a drug exposure of interest observed during the reporting period [20]. All ICSRs captured within VigiBase ${ }^{\circledR}$ from Zimbabwe collected from 1997 to 2018 were extracted and included in the analyses, as previously described by Masuka and Khoza [21]. The reported ADRs were coded using the Medical Dictionary for Regulatory Activities (MedDRA), version 21.0, at the PT level. All drugs were classified according to the anatomical therapeutic classification (ATC) system at level 1, and all reported medicines were considered in the analyses regardless of the causality assessment.

\section{Selection of cases and non-cases}

Cases were defined as ICSRs with the PT/ADR of interest, whereas non-cases (controls) were defined as all other ICSRs. The chosen HAART/ATT and HAART/cotrimoxazole combinations were considered the exposures of interest, and the remaining drugs were not considered exposures of interest. Positive and negative controls were used to investigate undetected biases in the study using known drug-drug combinations and their outcomes [20]. The known interaction between isoniazid and stavudine, leading to peripheral neuropathy, was used as a positive control, whereas the lack of known DDIs between tenofovir and cotrimoxazole was used as a negative control [22].

Drug-drug event combinations were selected from cited toxicity overlaps between HAART (ATC code J05AR) and ATT (J04AM) [15, 16]. Additional analyses were conducted for particular HAART combinations, such as nevirapinebased HAART or efavirenz-based HAART in combination with ATT for the same events. A similar approach was used for combinations of cotrimoxazole and HAART or ATT. These drug-drug event combinations were based on observations from our group's previous study [19]. Furthermore, related PTs were combined to form new identifier event terms as previously done by van Puijenbroek et al. [10]. Two ADRs of special interest (ADRSIs) were created for analysis as alternatives to the PT event levels [23, 24]: severe cutaneous adverse drug reaction (SCAR) comprised acute generalized exanthematous pustulosis, drug-induced hypersensitivity syndrome (DIHS), drug reaction with eosinophilia and systemic symptoms (DRESS), erythema multiforme, Stevens-Johnson syndrome (SJS), and toxic epidermal necrolysis (TEN) [25]. All selected drug-drug event combinations were initially reviewed against DDI information held in the freely available Liverpool HIV DDI interaction checker [22].

\section{Statistical analysis}

The detection of DDIs was based on the concept that when an ADR is reported more often for a combination of two drugs, $D_{1}$ and $D_{2}$ versus either $D_{1}$ or $D_{2}$ alone, then $D_{1}$ and $D_{2}$ interact when used in combination $[3,10]$. ADR reporting odds ratios (RORs) were calculated for each drug-drug event using a bivariate disproportionality analysis, represented by 
the $2 \times 2$ contingency table (Table 1 ) and formally indicated in Eqs. 1 and 2 [20, 26]:

$\mathrm{ROR}=\frac{a d}{b c}$

$95 \% \mathrm{CI}=\mathrm{e}^{\ln (\mathrm{ROR}) \pm 1.96 \sqrt{ }(1 / a+1 / b+1 / c+1 / d)}$,

where $a, b, c$, and $d$ are the respective frequencies indicated in Table 1 and CI is confidence interval.

Drug-drug event combinations for the concurrent use of $D_{1}$ and $D_{2}$ with a lower bound of the $95 \%$ confidence interval (CI) for the ROR $>1$ were considered SDRs [10, 20, 23]. An SDR was considered more likely to be due to a DDI if the 95\% CI for concomitant use was mutually exclusive of the two index groups [10]. Statistical analyses were conducted using the Statistical Package for Social Sciences (SPSS), version 16. The RORs and their associated 95\% CIs were calculated using an online statistical calculator (Select Statistical Services, Exeter, UK) [27]. In addition, all analyses were premised on a further assumption that the SRS data are representative of the event rate in the population using the medicines [9].

\section{Results}

A total of 4072 ICSRs were used in this analysis, of which 2560 and 540 were for HAART- and ATT-containing medications, respectively. The most commonly reported HAART combinations in the database were tenofovir/lamivudine/ efavirenz $(785 ; 30.66 \%)$, followed by stavudine/lamivudine/ nevirapine $(526 ; 20.55 \%)$, and tenofovir/lamivudine/nevirapine $(302 ; 11.80 \%)$ from 2560 HAART-containing ICSRs. Isoniazid and rifampicin were co-reported in 123 of the 540 ATT-containing ICSRs, and isoniazid was solely reported in 409 ICSRs. Cotrimoxazole was reported in 1052 ICSRs and co-reported with HAART in 870 ICSRs.

\section{Signals of disproportionate reporting}

Potential SDRs were observed for the PTs hepatotoxicity and jaundice with the concurrent use of HAART and ATT. This

Table $12 \times 2$ contingency table for the calculation of adverse drug reaction reporting odds ratios

\begin{tabular}{|lll|}
\hline Medication & ADR of interest "cases" & $\begin{array}{l}\text { Other ADR } \\
\text { "non-cases" }\end{array}$ \\
\hline Drug of interest & a & b \\
\hline Other drugs & c & d \\
\hline
\end{tabular}

$A D R$ adverse drug reaction was particularly observed for the concurrent use of HAART and isoniazid and of nevirapine-based HAART and isoniazid, as evidenced by the higher observed RORs. Concurrent use of HAART and rifampicin was also statically significant for both hepatotoxicity and jaundice, although the RORs were smaller, as shown in Table 2.

Potential SDRs were also observed for the ADRSI SCAR and the PTs SJS and rash, especially with the concurrent use of nevirapine-based HAART and cotrimoxazole. No similar, statistically significant interactions were observed for the concurrent use of efavirenz-based HAART and cotrimoxazole, as evidenced by RORs inclusive of 1 , as shown in Table 3 . The a priori selected positive and negative controls were confirmed as known signals and non-signals, respectively, as shown in Table 4.

\section{Discussion}

In the current study, we explored whether it was feasible to quantitatively detect potential DDIs using a relatively small SRS database. Poorly characterized DDIs involving antiretrovirals, ATT, and cotrimoxazole were chosen for the demonstration using an SRS database from a country with a high HIV and tuberculosis burden [28, 29]. ADRs are 7.4 times more common in patients with concurrent HIV and tuberculosis, whereas DDIs occur in up to $18 \%$ of patients with HIV [28, 30]. Using $2 \times 2$ contingency tables, we demonstrated that exploratory signal detection analyses are feasible within small databases, as previously discussed by Caster et al. [12]. Thus, timely quantitative signal detection can potentially be conducted using small databases from relatively underdeveloped pharmacovigilance systems, which will improve patient safety.

Several studies have indicated significant DDIs between HAART and ATT or antimicrobial drugs in Africa [13, 28, 29, 31]. In this study, we observed potential SDR for the hepatic system MedDRA PTs hepatotoxicity and jaundice associated with the concurrent use of nevirapine-based HAART and ATT or isoniazid prophylactic therapy (IPT). These observations strengthen the clinically and qualitatively observed signals of possible DDIs between nevirapine-based HAART and IPT [19]. These findings have also recently been highlighted in the paediatric population within a Zimbabwean specialist HIV clinic setting [32]. Previous studies indicated minimal hepatotoxicity risk with the concurrent use of nevirapine-based HAART and isoniazid and/or ATT [33]. However, some of these studies were of short duration or were largely composed of participants not receiving HAART [33]. Isoniazid appears to cause a pharmacokinetic DDI with nevirapine, resulting in elevated serum concentrations of nevirapine [34], possibly explaining the current observations. 
Table 2 Adverse event reporting odds ratios depicting the occurrence of hepatotoxicity and jaundice

\begin{tabular}{|c|c|c|c|c|c|c|}
\hline \multirow[t]{2}{*}{ Exposure } & \multicolumn{3}{|c|}{ Hepatotoxicity } & \multicolumn{3}{|c|}{ Jaundice } \\
\hline & Cases & Controls & ROR $(95 \% \mathrm{CI})$ & Cases & Controls & ROR $(95 \% \mathrm{CI})$ \\
\hline \multicolumn{7}{|l|}{ J05AR-J04AM } \\
\hline Neither J05AR nor J04AM & 1 & 1131 & 1 & 3 & 1129 & 1 \\
\hline J05AR & 10 & 2389 & $4.73(0.61-37.03)$ & 30 & 2369 & $4.77(1.45-15.65)$ \\
\hline J04AM & 5 & 374 & $15.12(1.76-129.83)$ & 15 & 364 & $15.51(4.46-53.87)$ \\
\hline Concomitant J05AR + J04AM & 6 & 155 & $43.78(5.24-366.08)$ & 24 & 137 & $65.93(19.60-221.81)$ \\
\hline \multicolumn{7}{|l|}{ J05AR-INH } \\
\hline Neither J05AR nor INH & 1 & 1136 & 1 & 4 & 1133 & 1 \\
\hline J05AR & 10 & 2392 & $4.75(0.61-37.14)$ & 30 & 2372 & $3.58(1.2-10.19)$ \\
\hline INH & 5 & 369 & $15.39(1.79-132.18)$ & 14 & 360 & $11.02(3.60-33.68)$ \\
\hline Concomitant J05AR + INH & 6 & 152 & $44.84(5.36-374.99)$ & 24 & 134 & $50.73(17.34-148.43)$ \\
\hline \multicolumn{7}{|l|}{ J05AR-RIF } \\
\hline Neither J05AR nor RIF & 4 & 1400 & 1 & 10 & 1434 & 1 \\
\hline J05AR & 14 & 2487 & $1.97(0.65-6.00)$ & 50 & 2451 & $2.93(1.48-5.79)$ \\
\hline RIF & 2 & 65 & $10.77(1.94-59.87)$ & 8 & 59 & $19.44(7.40-51.06)$ \\
\hline Concomitant J05AR + RIF & 2 & 57 & $12.28(2.20-68.44)$ & 4 & 55 & $10.43(3.17-34.29)$ \\
\hline \multicolumn{7}{|l|}{ EFV-INH } \\
\hline Neither EFV nor INH & 9 & 2545 & 1 & 24 & 2530 & 1 \\
\hline EFV & 2 & 983 & $0.58(0.12-2.67)$ & 10 & 975 & $1.08(0.52-2.27)$ \\
\hline INH & 9 & 241 & $10.56(4.15-26.85)$ & 32 & 218 & $15.47(8.95-26.74)$ \\
\hline Concomitant EFV + INH & 2 & 280 & $2.02(0.43-9.39)$ & 6 & 276 & $2.29(0.93-5.65)$ \\
\hline \multicolumn{7}{|l|}{ NVP-INH } \\
\hline Neither NVP nor INH & 4 & 2449 & 1 & 23 & 2430 & 1 \\
\hline NVP & 7 & 1079 & $3.97(1.16-13.60)$ & 11 & 1075 & $1.08(0.53-2.23)$ \\
\hline INH & 6 & 435 & $8.44(2.37-30.05)$ & 19 & 422 & $4.76(2.57-8.81)$ \\
\hline Concomitant NVP + INH & 5 & 86 & $35.60(9.39-134.89)$ & 19 & 72 & $27.88(14.54-53.47)$ \\
\hline
\end{tabular}

$E F V$ efavirenz, INH isoniazid, JO4AM antimycobacterial drugs, J05AR antiretroviral drug class, NVP nevirapine, RIF rifampicin, ROR reporting odds ratio

Similarly, potential SDRs were observed for the coadministration of nevirapine-based HAART and cotrimoxazole for the PTs rash and SJS and the medically important term, SCAR. In one case-control study, concurrent nevirapine-based HAART and ATT was associated with an increased risk for severe cutaneous ADRs, including SJS and DIHS/DRESS [35]. The authors postulated that this observation may be due to concomitantly administered cotrimoxazole, though this hypothesis was not analysed [35]. SCAR, particularly SJS/TEN secondary to concurrent use of nevirapine-based HAART and cotrimoxazole prophylactic therapy, has been observed in a similar Sub-Sahara African population [36]. Concurrent use of nevirapine-based HAART and cotrimoxazole has been associated with hepatotoxicity. Furthermore, increased aminotransferase levels were associated with rashes [37].

The major strengths of this validated, frequentist disproportionality analysis include reasonable sensitivity and easy applicability and interpretability [20]. Non-selective underreporting of a drug or an ADR does not influence the value of the ROR compared with the population experiencing the ADR [26]. Furthermore, this study used de-duplicated ADRs not reported as DDIs, which potentially enhances the study's internal validity [19]. Stratification and/or subgrouping minimized selection and competition biases [20]. The use of "control drug-drug events" ruled out undetected biases since it verified known and unknown signals for positive and negative controls, respectively $[9,20]$.

The risk of the observed signals being spurious might be minimal [12]; however, there are a multitude of significant limitations in this study. First, the small database and the incomplete reporting of ADRs and/or concurrent medications limit the reliability of the SRS-derived data for quantitative signal detection. Second, this small database also limited the precision and the magnitude of the observed potential SDRs as indicated by the wide 95\% CIs [38]. For instance, it is difficult to know with certainty whether patients were receiving IPT or ATT, as ICSRs frequently lack data on concomitant medications, amongst other parameters [20]. Thus, the observed RORs are only indicative 
Table 3 Adverse event reporting odds ratios depicting the occurrence of rash, Steven-Johnson syndrome, and SCAR

\begin{tabular}{|c|c|c|c|c|c|c|c|c|c|}
\hline \multirow[t]{2}{*}{ Exposure } & \multicolumn{3}{|l|}{ Rash } & \multicolumn{3}{|c|}{ Steven-Johnson syndrome } & \multicolumn{3}{|l|}{ SCAR } \\
\hline & Cases & Controls & ROR (95\% CI) & Cases & Controls & ROR (95\% CI) & Cases & Controls & ROR (95\% CI) \\
\hline \multicolumn{10}{|l|}{ J05AR-CTX } \\
\hline Neither J05AR nor CTX & 213 & 1116 & 1 & 6 & 1323 & 1 & 16 & 1313 & 1 \\
\hline J05AR & 232 & 1458 & $0.83(0.68-1.02)$ & 47 & 1643 & $6.31(2.69-14.80)$ & 55 & 1635 & $2.76(1.57-4.84)$ \\
\hline CTX & 46 & 136 & $1.77(1.27-2.55)$ & 10 & 172 & $12.82(4.60-35.71)$ & 14 & 168 & $6.84(3.28-14.26)$ \\
\hline $\begin{array}{l}\text { Concomitant } \\
\text { J05AR + CTX }\end{array}$ & 147 & 723 & $1.07(0.85-1.37)$ & 46 & 824 & $12.31(5.23-28.95)$ & 55 & 815 & $5.54(3.15-9.73)$ \\
\hline \multicolumn{10}{|l|}{ EFV-CTX } \\
\hline Neither EFV nor CTX & 343 & 1842 & 1 & 48 & 2137 & 1 & 64 & 2121 & 1 \\
\hline EFV & 102 & 732 & $0.75(0.59-0.95)$ & 5 & 829 & $0.27(0.11-0.68)$ & 7 & 827 & $0.28(0.13-0.61)$ \\
\hline CTX & 128 & 491 & $1.40(1.12-1.76)$ & 49 & 570 & $3.83(2.54-5.76)$ & 57 & 562 & $3.36(2.32-4.86)$ \\
\hline Concomitant EFV + CTX & 65 & 368 & $0.95(0.71-1.26)$ & 7 & 426 & $0.73(0.33-1.63)$ & 12 & 421 & $0.94(0.51-1.77)$ \\
\hline \multicolumn{10}{|l|}{ NVP-CTX } \\
\hline Neither NVP nor CTX & 289 & 1979 & 1 & 9 & 2259 & 1 & 19 & 2249 & 1 \\
\hline NVP & 156 & 595 & $1.75(1.41-2.17)$ & 44 & 707 & $15.62(7.59-32.16)$ & 52 & 699 & $8.81(5.17-14.99)$ \\
\hline CTX & 88 & 538 & $1.09(0.84-1.41)$ & 12 & 614 & $4.91(2.06-11.70)$ & 18 & 608 & $3.5(1.82-6.72)$ \\
\hline Concomitant NVP+CTX & 105 & 321 & $2.18(1.69-2.81)$ & 44 & 382 & $28.91(14.00-59.70)$ & 51 & 375 & $16.1(9.40-27.57)$ \\
\hline
\end{tabular}

$C T X$ cotrimoxazole, EFV efavirenz, JO5AR antiretroviral drug class, $N V P$ nevirapine, ROR reporting odds ratio, SCAR severe cutaneous adverse drug reaction

\section{Table 4 Adverse event reporting odds ratios for positive and nega-} tive controls

\begin{tabular}{|lrrll|}
\hline Exposure & Cases & Controls & ROR $(95 \%$ CI $)$ \\
\hline Anaemia TDF-CTX & & & \\
\hline Neither TDF nor CTX & 29 & 1927 & 1 \\
\hline TDF & 19 & 1044 & $1.21(0.67-2.17)$ \\
CTX & 16 & 425 & $2.50(1.35-4.65)$ \\
\hline Concomitant & 17 & 594 & $1.90(1.04-3.49)$ \\
$\quad$ TDF+CTX & & & \\
\hline Peripheral neuropathy D4T-INH & & \\
\hline Neither D4T nor INH & 22 & 2870 & 1 \\
\hline D4T & 227 & 420 & $70.51(44.98-110.52)$ \\
\hline INH & 25 & 470 & $6.94(3.88-12.41)$ \\
\hline Concomitant use & 21 & 16 & $171.22(78.97-371.25)$ \\
$\quad$ D4T + INH & & & \\
\hline
\end{tabular}

CTX cotrimoxazole, D4T stavudine, INH isoniazid, ROR reporting odds ratio, $T D F$ tenofovir

for all DDIs with concurrent isoniazid IPT or ATT. The observed RORs may have been overestimated because of selection bias, particularly indication bias, because we did not stratify the analyses according to therapeutic class [20].

In addition to under-reporting, competition, selection, and surveillance biases may limit the interpretability of the presented results $[11,20]$. Clinical limitations such as poor reporting of concomitant alcohol and/or herbal medications and assumption of $100 \%$ drug adherence and standardized dosing further limit the study's validity.
The described approach may only be useful for synergistic rather than antagonistic interactions [11]. Moreover, our approach limited the study because we did not test for interaction effects or correct for covariates as done in a logistic regression modelling approach $[10,20]$. However, a review of the Liverpool HAART drug interaction checker did not indicate some of the SDRs observed in our study. Similarly, the University of California, San Francisco HIV InSite antiretroviral database does not indicate any of the observed SDRs [39].

In conclusion, quantitative signal detection analyses for potential, unrecognized, or poorly characterized SDR of DDIs is feasible within relatively small SRS databases. This is especially important for DDIs resulting from HAART and/or ATT in Sub-Sahara Africa. The region has a high background rate of comorbid HIV and tuberculosis and use of standardized therapy and fixed-dose HAART and/ATT drug combinations, lacks affordable alternative medicines, and has significantly weak drug safety monitoring systems [14]. The detection of SDRs does not imply a causal relationship; it only generates hypotheses, possibly just at par with case reports and/or series $[9,10,41]$. The clinical validity of the raised hypotheses must be further reviewed and evaluated by subject matter experts [41]. Further assessment of the observed potential SDRs using larger datasets and/or controlled epidemiologic studies is needed to verify findings from disproportionalty analyses $[40,41]$. 


\section{Take home messages}

- Quantitative disproportionate analyses for drug-drug interactions are feasible in relatively small spontaneous adverse drug reaction reporting systems.

- Concomitant use of antiretrovirals and isoniazid potentially increases the risk of hepatotoxicity.

- Concomitant use of nevirapine-based antiretrovirals and cotrimoxazole potentially increases the risk of cutaneous adverse drug reactions.

\section{Declarations}

Funding No sources of funding were used to conduct this study or prepare this manuscript.

Conflict of interest Josiah Tatenda Masuka, Anisa Mosam, and Star Khoza have no conflicts of interest that are directly relevant to the content of this article.

Ethics approval Ethical approval for the study was waived by the Medical Research Council of Zimbabwe (MRCZ Ref: MRCZ/E/207) in view of the retrospective nature of the study. The study used routinely collected depersonalized and de-identified data.

Consent to participate Not applicable.

Availability of data and material The data that support the findings of this study are available from the corresponding author upon reasonable request.

Code availability Not applicable.

Author contributions JTM conceived and designed the study and drafted the manuscript. JTM and SK performed the research and analysed the data. JTM, AM, and SK reviewed and approved the final manuscript version.

\section{References}

1. Parmentier Y, Bossant M, Bertrand M, et al. In vitro studies of drug metabolism. In: Taylor JB, Triggle JD, editors. Comprehensive medicinal chemistry II. 2nd ed. Cambridge: Academic Press; 2007. p. 231-57.

2. Huang J, Niu C, Green C, et al. Systematic prediction of pharmacodynamic drug-drug interactions through protein-proteininteraction network. PLoS Comput Biol. 2013;9(3):e1002998.

3. Heba I, Amany A, Ahmed S, et al. Novel data-mining methodologies for detecting drug-drug interactions: a review of pharmacovigilance literature. Adv Environ Sci Dev Chem. 2014;20:301-14.

4. Strandell J, Bate A, Lindquist M, et al. Drug-drug interactions: a preventable patient safety issue? Br J Clin Pharmacol. 2008;65(1):144-6.
5. Huang S, Temple R, Throckmorton D, et al. Drug interaction studies: study design, data analysis, and implications for dosing and labeling. Clin Pharm Ther. 2007;81(2):298-304.

6. Dechanont S, Maphanta S, Butthum B, et al. Hospital admissions/ visits associated with drug-drug interactions: a systematic review and meta-analysis. Pharmacol Drug Saf. 2014;23:489-97.

7. Snyder B, Polasek T, Doogue M. Drug interactions: principles and practice. Austr Prescr. 2012;35(3):85-8.

8. Thakrar B, Grundschober S, Doessegger L. Detecting signals of drug-drug interactions in a spontaneous reports database. Br J Clin Pharmacol. 2007;64(4):489-95.

9. van Puijenbroek E, Egberts A, Heerdink E, et al. Detecting drugdrug interactions using a database for spontaneous adverse drug reactions: an example with diuretics and non-steroidal anti-inflammatory drugs. Eur J Clin Pharmacol. 2000;56(9-10):733-8.

10. van Puijenbroek E, Egberts A, Meyboom R, et al. Signalling possible drug-drug interactions in a spontaneous reporting system: delay of withdrawal bleeding during concomitant use of oral contraceptives and itraconazole. Br J Clin Pharmacol. 1999;47:689-93.

11. Noguchi Y, Tachi T, Teramachi H. Review of statistical methodologies for detecting drug-drug interactions using spontaneous reporting systems. Front Pharmacol. 2019;10:1319.

12. Caster O, Aoki Y, Gattepaille L, et al. Disproportionality analysis for pharmacovigilance signal detection in small databases or subsets: recommendations for limiting false-positive associations. Drug Saf. 2020;43:479-87.

13. Semvua H, Kibiki G, Kisanga E, et al. Pharmacological interactions between rifampicin and antiretroviral drugs: challenges and research priorities for resource-limited settings. Ther Drug Monit. 2015;37(1):22-32.

14. McIlleron $\mathrm{H}, \mathrm{Khoo} \mathrm{SH}$. Interactions between antituberculosis and antiretroviral agents. Prog Respir Res. 2011;40:191-202.

15. McIlleron H, Meintjes G, Burman W, et al. Complications of antiretroviral therapy in patients with tuberculosis: drug interactions, toxicity, and immune reconstitution inflammatory syndrome. J Infect Dis. 2007;196(Suppl 1):S63-S75.

16. Di Perri G, Aguilar Marucco D, Mondo A, et al. Drug-drug interactions and tolerance in combining antituberculosis and antiretroviral therapy. Expert Opin Drug Saf. 2005;4(5):821-36.

17. Maartens G, Boffito M, Flexner C. Compatibility of next-generation first-line antiretrovirals with rifampicin-based antituberculosis therapy in resource limited settings. Curr Opin HIV AIDS 2017;12(4):355-8.

18. Lalloo U. Efavirenz and nevirapine interactions with rifampicin: resolving the dilemmas? Clin Infect Dis. 2009;48(12):1760-2.

19. Masuka J, Chipangura P, Nyambayo P, et al. A comparison of adverse drug reaction profiles in patients on antiretroviral and antitubercular treatment in Zimbabwe. Clin Drug Investig. 2018;38(1):9-17.

20. Faillie J. Case-non-case studies: principle, methods, bias andinterpretation. Therapie. 2019;74:225-32.

21. Masuka J, Khoza S. An analysis of the trends, characteristics, scope and performance of the Zimbabwean pharmacovigilance reporting scheme. Pharmacol Res Perspect. 2020;8(5):e00657.

22. University of Liverpool Group. HIV drug interactions. University of Liverpool. https://www.hiv-druginteractions.org/checker. Accessed 14 Mar 2019.

23. van Puijenbroek E, van Grootheest K, Diemont W, et al. Determinants of signal selection in a sponrtaneous reportingsystemfor adverse drug reactions. Br J Clin Pharmacol. 2001;52(5):579-86.

24. The Development of the Safety Update Report (DSUR): harmonising the format and content for periodic safety reporting during clinical trials: Report of CIOMS Working Group VII. 2007. 
25. Duong $\mathrm{T}$, Valeyrie-Allanore $\mathrm{L}$, Wolkenstein $\mathrm{P}$, et al. Severe cutaneous adverse drug reactions to drugs. Lancet. 2017;390(10106):1996-2011.

26. Van Puijenbroek E, Bate A, Leufkens H, et al. A comparison of measures of disproportionality for signal detection in spontaneous reporting systems for adverse drug reactions. Pharmacol Drug Saf. 2002;11(1):3-10.

27. Select Statistical Services. Odds ratio-confidence interval. https ://select-statistics.co.uk/calculators/confidence-interval-calculator -odds-ratio/. Accessed 14 June 2020.

28. Seden K, Merry C, Hewson R, et al. Prevalence and type of drugdrug interactions involving ART in patients attending a specialist HIV outpatient clinic in Kampala, Uganda. J Antimicrob Chemother. 2015;70(12):3317-22.

29. Kigen G, Kimaiyo S, Nyandiko W, et al. Prevalence of potential drug-drug interactions involving antiretroviral drugs in a large Kenyan cohort. PLoS ONE. 2011;6(2):e16800.

30. Modayil R, Harugeri A, Parthasarathi G, et al. Adverse drug reactions to antiretroviral (ART): an experience of spontaneous reporting and intensive monitoring from ART centre in India. Pharmacol Drug Saf. 2010;19:247-55.

31. Seden K, Gibbons S, Marzolini C, et al. Development of an evidence evaluation and synthesis system for drug-drug interactions, and its application to a systematic review of HIV and malaria co-infection. PLoS ONE. 2017;12(3):e0173509.

32. Mudzviti T, Shamu T, Chimbetete C, et al. Tolerability of isoniazid prevention therapy in an HIV-infected cohort of paediatric and adolescent patients on antiretroviral therapy from a resourcelimited setting: a retrospective cohort study. Drugs Real World Outcomes. 2019;6(1):37-42.

33. Tedla Z, Nyirenda S, Peeler C, et al. Isoniazid-associated hepatitis and antiretroviral drugs during tuberculosis prophylaxis in
HIV infected adults in Botswana. Am J Respir Crit Care Med. 2010;182(2):278-85.

34. Decloedt E, Mwansa-Kambafwile J, van Der Walt, et al. The pharmacokinetics of nevirapine when given with isoniazid in South African HIV-infected individuals. Int J Tuberc Lung Dis. 2013;17(3):333-5.

35. Stewart A, Lehoenya R, Boulle A, et al. Severe antiretroviral-associated skin reactions in South African patients: a case series and case-control analysis. Pharmacol Drug Saf. 2016;25(11):1313-9.

36. Oshikoya K, Ogunyinka I, Ogar C, et al. Severe cutaneous adverse drug reactions manifesting as Stevens-Johnson syndrome and toxic epidermal necrolysis reported to the national pharmacovigilance center in Nigeria: a databse review from 2004-2017. Ther Adv Drug Saf. 2020;11:2042098620905998.

37. Tseng $\mathrm{Y}$, Yang $\mathrm{C}$, Chang $\mathrm{S}$, et al. Incidence and risk factors of skin rashes and hepatotoxicity in HIV-infected patients receiving nevirapine-containing combination antiretroviral therapy in Taiwan. Int J Inf Dis. 2014;29:12-7.

38. Sim J, Reid N. Statistical inference by confidence intervals: Issues of interpretation and utilization. Phys Ther. 1999;79(2):186-95.

39. Center for HIV Information (CHI) UCSF. UCSF HIV Insite database of antiretroviral drug interactions. UCSF, California. 2019. https://arv.ucsf.edu/insite?page=a. Accessed 17 Aug 2020.

40. Hennessy S. Disproportionality analyses of spontaneous reports. Pharmacol Drug Saf. 2004;13:503-4.

41. Norén G, Sundberg R, Bate A, et al. A statistical methodology for drug-drug interaction surveillance. Stat Med. 2008;27(16):3057-70. 